\title{
Imposing spatio-temporal support in magnetic resonance angiographic imaging
}

\author{
Philip J. Bones ${ }^{a}$, Bahareh Vafadar ${ }^{a}$, Richard $\mathrm{Watts}^{b}$ and Bing $\mathrm{Wu}^{c}$ \\ ${ }^{a}$ Computational Imaging Group, Department of Electrical \& Computer Engineering, \\ University of Canterbury, Christchurch, New Zealand. \\ ${ }^{b}$ Department of Physics \& Astronomy, University of Canterbury, Christchurch, New Zealand. \\ ${ }^{c}$ Brain Imaging and Analysis Center, School of Medicine, Duke University, Durham, North \\ Carolina, U.S.A.
}

\begin{abstract}
A method to improve time resolution in 3D contrast-enhanced magnetic resonance angiography (CE-MRA) is proposed. A temporal basis based on prior knowledge of the contrast flow dynamics is applied to a sequence of image reconstructions.

In CE-MRA a contrast agent (gadolinium) is injected into a peripheral vein and MR data is acquired as the agent arrives in the arteries and then the veins of the region of clinical interest. The acquisition extends over several minutes. Information is effectively measured in 3D k-space (spatial frequency space) one line at-atime. That line may be along a Cartesian grid line in $\mathrm{k}$-space, a radial line or a spiral trajectory. A complete acquisition comprises many such lines but in order to improve temporal resolution, reconstructions are made from only partial sets of k-space data. By imposing a basis for the temporal changes, based on prior expectation of the smoothness of the changes in contrast concentration with time, it is demonstrated that a significant reduction in artifacts caused by the under-sampling of k-space can be achieved. The basis is formed from a set of gamma variate functions. Results are presented for a simulated set of 2D spiral-sampled CE-MRA data.
\end{abstract}

\section{INTRODUCTION}

In magnetic resonance imaging (MRI), information is effectively measured in 3D k-space (spatial frequency space) one line at-a-time. That line may be along a Cartesian grid line in k-space, a radial line or a spiral trajectory. A complete acquisition comprises many such lines but in order to improve temporal resolution, reconstructions are often made from partial sets of k-space data. In parallel MRI, multiple receiver coils are used so that several signals are recorded in parallel; the different sensitivity functions associated with the coils allow images to be reconstructed from fewer measurements than would otherwise be the case. ${ }^{1-3}$ However, the significant time needed to acquire data in MRI remains a significant problem. While techniques such as fast spin echo (FSE) and/or various non-Cartesian sampling strategies can be used to acquire image data relatively rapidly, the acquisition time for a full set of data is still normally longer than one minute. Some acquisition sequences take many minutes to complete. When MRI is employed to assess the state of the circulation with the aid of injected contrast material, the method is called contrast-enhanced magnetic resonance angiography (CE-MRA). The contrast material is a compound containing gadolinium (Gd), which alters the T1 property of the blood with which it mixes and differentiates it from the surrounding tissue. The contrast material is injected as a 'bolus', often by means of a powered syringe, usually into a peripheral vein. ${ }^{4}$ Some seconds later it arrives in the arteries within the region being imaged, the 'field-of-view' (FOV). In CE-MRA it is important to get sufficient time resolution to be able to observe the arrival and flow of the contrast material through the vessels within the FOV. A great deal of attention has therefore been directed at developing methods of acquiring and processing MR data to achieve the higher time resolution. This paper reports our efforts to utilize temporal basis functions for this purpose.

Further author information:

Philip J. Bones: E-mail: phil.bones@canterbury.ac.nz, Telephone: +64 33642987 ext. 7275 
MRA has become an accepted standard for evaluation for renovascular and peripheral vascular disease. ${ }^{4}$ The fact that it is noninvasive, compared to some other angiographic techniques which require use of ionizing radiation or the insertion of a catheter, is a particularly strong point in its favour. Not all MRA methods require contrast material: the time-of-flight (TOF) method can be used in cases where injection of Gd is contraindicated or when repeated studies are required. ${ }^{4}$ However, it is generally considered to be inferior to CE-MRA in terms of diagnostic quality. ${ }^{4,5}$ Only CE-MRA is considered here.

A number of authors have previously attempted to introduce a temporal basis into medical image reconstruction. The majority of the work to date has been directed at cardiac imaging, where the approximately periodic nature of the motion and the availability of an independent synchronous signal, the electrocardiogram, can be exploited. ${ }^{6-8}$ While the cardiac-related work helps to illustrate the potential for incorporating temporal constraints into the imaging process, it cannot be directly applied to the CE-MRA case. The effect of k-space sampling order on CE-MRA has also been studied. ${ }^{5,9}$ It was found that the best quality images were achieved when the center of k-space was sampled at the time when the peak concentration of contrast material reached the region of interest. The difficulty is that it is often desirable to observe the complete vascular system in a region and during both the arterial and venous phases, ${ }^{5}$ therefore it is desirable to have repeated rapid re-sampling of the center of k-space. Prince et al developed methods to automatically detect the arrival of the contrast material bolus at a particular point before commencing data acquisition. ${ }^{10}$ While this helped to reduce the need for careful synchronisation of the contrast injection with the collection of data, it did not in itself assist with achieving a higher temporal resolution. Korosec et al introduced 3D TRICKS (time-resolved imaging of contrast kinetics) in which a high sampling density was applied in the central area of k-space and temporal interpolation was applied within k-space. ${ }^{11}$ There does not appear to be any report of research into applying a particular temporal basis for CE-MRA.

In the paper, we review the methods used for parallel MRI and describe an algorithm we have developed for deriving a small set of temporal basis functions. We present some preliminary results which demonstrate the use of the temporal basis on a set of simulated data for spiral trajectory CE-MRA data acquisition. We then discuss how the method could be applied more widely.

\section{BACKGROUND}

\subsection{Parallel MRI}

In parallel MRI, an array of receiver coils is used to acquire simultaneous recordings. Since the k-space sampling trajectory is controlled by the gradient fields and excitation sequence, all coils receive information representing the same location in k-space. However each coil has a different sensitivity variation within the FOV because of the coil's different physical orientation and location. Methods such as SMASH (SiMultaneous Acquisition of Spatial Harmonics) ${ }^{1}$ and SENSE (SENSitivity Encoding) ${ }^{2}$ are designed to exploit the coil sensitivity variation.

SMASH uses linear combinations of the receiver coils signals to synthesize sinusoidal variation across the FOV and to thus effectively extend the k-space coverage associated with any k-space site measured by the excitation sequence. ${ }^{1}$ In this manner, fewer sequences are required to achieve Nyquist sampling in k-space and thus to form an image. SMASH and its derivatives are referred to as "k-space methods" for parallel MRI. ${ }^{3}$

In SENSE the signals from each coil are used to form an image, i.e., one image for each coil. In order to speed up the acquisition, fewer samples are made in k-space than would be adequate to provide an un-aliased image. The knowledge of the coil sensitivity variation is then used to reduce the aliasing. ${ }^{2}$ SENSE and similar methods are referred to as "image space methods" for parallel MRI. ${ }^{3}$

\subsection{Data acquisition}

The emphasis in parallel MRI to date has been on k-space sampling strategies which lead to sampling on the Cartesian grid. This suits SMASH-like methods since the synthesis of spatial harmonics straightforwardly can be used to recover k-space samples which are positioned at fixed offsets from measured points. It also suits SENSE-like methods, since block-based sampling of k-space allows the recovery problem to be partitioned into a large number of smaller problems. ${ }^{12-15}$ However, for achieving the high temporal resolution required in CE-MRA or other applications of MRI associated with movement, such as cardiovascular imaging, radial or spiral sampling 
trajectories may have advantages. ${ }^{16}$ Spiral k-space trajectories have been modeled in the simulations presented below.

Knowledge of the coil sensitivity variation across the FOV is central to the use of parallel MRI methods, so some form of calibration is required. ${ }^{3}$ One approach is to place a homogeneous spherical phantom within the scanner and to record a set of volume data. In many cases, however, in vivo calibration is performed, ${ }^{3}$ whereby a data acquisition sequence of the actual volume to be imaged is taken without any contrast being injected. This may ba separate acquisition, or part of the main acquisition sequence completed before the injection. Since the signal from each location in the object should be the same, the only differences in signal (both amplitude and phase) received by the various coils should be due to the differences in their sensitivity (plus a relatively minor contribution from electronic noise). To save time, a calibration sequence is often recorded at lower resolution than the main imaging sequence. ${ }^{3}$

\subsection{Modeling temporal variation}

In contrast-enhanced MRA a bolus of gadolinium-containing compound is injected into a peripheral vein. The injected material diffuses within the bloodstream as it is propelled to the right side of the heart, through the lungs, through the left side of the heart and then finally to the site of interest. The diffusion within the moving bloodstream is such that the concentration of contrast material, observed at some downstream point, rises from zero to a peak and then decays somewhat more slowly than it rose. If monitoring continues, it may be possible to see a later recirculation phase, but attention is normally directed to the first pass of the contrast material. A number of authors have analysed the shape of the contrast-versus-time curve and concluded that it approximates to a gamma variate function ${ }^{17,18}$ (neglecting the recirculation, if present). Ample experimental evidence exists for the applicability of the gamma variate function to studies involving the use of a marker substance. ${ }^{19-21}$

In its most common form the gamma variate function can be expressed as

$$
y(t)=A\left(t-t_{0}\right)^{\alpha} \exp \left(-\left(t-t_{0}\right) / \beta\right), \quad t \geq t_{0},
$$

where $t_{0}$ is the point at which the function commences and $\alpha$ and $\beta$ are real parameters. However Madsen ${ }^{18}$ showed how it can be expressed in a more convenient form:

$$
y(\tau)=y_{\max } \tau^{\alpha} \exp (\alpha(1-\tau)),
$$

where $\tau=\left(t-t_{0}\right) /\left(t_{\max }-t_{0}\right), t_{\max }$ is the time at which the function peaks and $y_{\max }$ is the amplitude at that time. The shape of the gamma variate function in the form of Eq. 2 is controlled by the single parameter, $\alpha$.

\subsection{Forming a temporal basis}

Within a sequence of MRA images of a fixed FOV, the expectation is that different pixels (or voxels if the images are 3D) will have distinctly different temporal variation throughout the sequence. Firstly, those associated with areas free of significant bloodflow will have little temporal variation other than the inevitable signal noise and the artifacts likely to be caused if the sampling density in k-space is below the Nyquist limit. Those associated with arteries will exhibit a relatively early and rapidly rising contrast pulse. Those associated with the venous drainage will exhibit a relatively later and more slowly rising pulse with lower peak amplitude than for the arterial pixels (voxels). The time of onset will depend on the relative distance from the FOV entry point for the bloodflow to the point of observation and the timing of the injection relative to the start of the data acquisition.

While the gamma variate function provides a very useful model for the expected intensity variation in those parts of the image where blood vessels lie and therefore through which blood and contrast flows, the function has several parameters controlling the temporal variation, i.e., $t_{0}, t_{\max }$ and $\alpha$, as presented above in Subsection 2.3 . Our approach to constraining the temporal variation is to form a set of basis functions a linear combination of which can accurately represent the range of responses expected within the image. We have employed the Karhunen-Loeve transform (KLT) which is known to provide an optimal basis under certain conditions. ${ }^{22}$

Our algorithm can be summarized as follows: 
1. A set of gamma variate functions, $K$ in number, is formed with different randomly chosen characteristics in terms of Eq. 2 and the parameters $t_{0}, t_{\max }$, and $\alpha$; the set are stored in a matrix $G$, dimensioned $K \times N$, where $N$ is the number of time samples over which the MRA sequence is to be modeled. In practice, $N$ depends on the characteristics of the data acquisition sequences employed and the time resolution sought.

2. The KLT is applied to the set by computing the eigenvectors of the covariance matrix of $G, G G^{T}$.

3. The $\hat{K}$ eigenvectors associated with the largest eigenvalues of $G G^{T}$ are chosen as the temporal basis functions. Each eigenvector has $N$ elements and represents a temporal basis function.

4. The sequence of intensity values for all pixels (voxels) in the MRA image sequence are projected onto the temporal basis. In this manner the $N$ independent values associated with the time variation for each pixel is mapped onto $\hat{K}$ coefficients, where $\hat{K}<N$.

Since the number of temporal basis functions retained in the algorithm, $\hat{K}$, is chosen to be less than $N$, there is clearly compression occurring, which could be advantageous. However, the main benefit of applying the temporal basis is in the suppression of artifacts, since they are very unlikely to be correlated with the temporal basis functions. The simulations presented in the next section demonstrate how the algorithm achieves a significant improvement in image sequence quality.

\section{SIMULATIONS}

\subsection{The "Reconstruction Challenge"}

As part of the annual conference of the International Society for Magnetic Resonance Imaging in Medicine (ISMRM) in 2010, a competition was run to see which group could generate the best reconstructions from several sets of data. One of the sets of data, given the name "Need for speed" in the competition, was a sequence of spiral-sampled k-space data for a parallel MRI acquisition. In fact, the data was simulated by re-sampling an image sequence collected by X-ray digital subtraction angiography, but it closely resembled the sort of data expected for an MRI study. It provided a useful vehicle for testing our algorithm.

Data were simulated using a frontal projection X-ray sequence of a contrast bolus injection in the left vertebral artery of a patient with an arteriovenous malformation (AVM) in the brain. X-ray data were collected 3 frames per second, for a total of 10 seconds (31 collected frames) which span wash-in to wash-out. These were linearly interpolated in time between frames to create a total of 200 temporal images, each with 512x512 resolution. Coil sensitivity maps derived from an axial slice through a water phantom using an 8-channel head coil were superimposed on the image to create 8 "coil" images. Independent noise was added to each channel. After Fourier transformation, the k-space data were re-sampled over 200 trajectories, one per frame, each with 2000 points.

In Fig. 1(a) is shown the first two consecutive spiral trajectories for sampling in k-space. The center of k-space represents zero spatial frequency and sampling occurs out to radius 0.5 on the scale shown, corresponding to an image FOV of $512 \times 512$. The series of spiral trajectories repeats every 13 spirals and the 13 are uniformly angularly spaced over 360deg. Note however that each spiral is rotated by approximately 111deg from its predecessor in acquisition order. In Fig. 1(b) is shown a reconstructed image which was formed from all of the k-space NFS data for all 8 coils over the acquisition sequence (RMS over the coils, summed over time).

\subsection{Reconstruction of images}

Each spiral trajectory comprises 2000 samples, commencing at the center of $\mathrm{k}$-space. In principle an image can be reconstructed from any number of consecutive spirals: the fewer the number of spirals used, the better the time resolution. We chose to use 5 consecutive spirals to reconstruct each frame, representing a frame rate of 4 frames / sec. 5 spirals represents a total of $10000 \mathrm{k}$-space samples, compared to approximately $\pi 256^{2}=205890$ samples in order to fully sample the cartesian grid within a circular region. There is therefore a high degree of under-sampling in k-space. A number of methods have been used to re-grid under-sampled k-space data onto a Cartesian grid. However, the sparsity of the data in this case makes those methods relatively ineffective. Our approach instead has been to perform a simple nearest-neighbour allocation of samples onto grid points for 


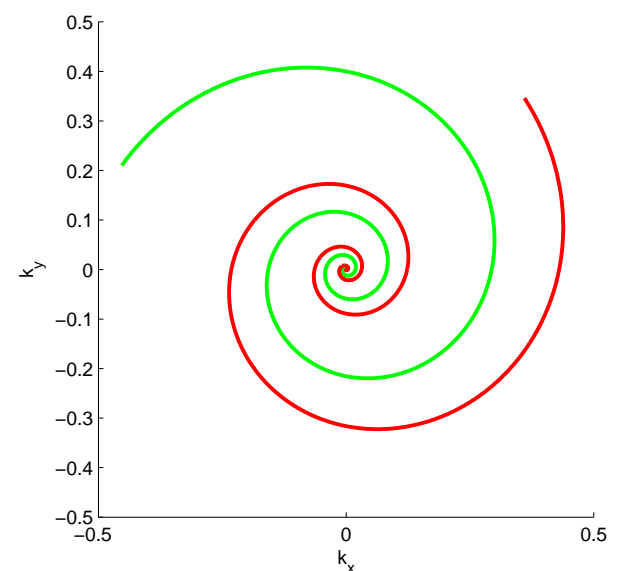

(a)

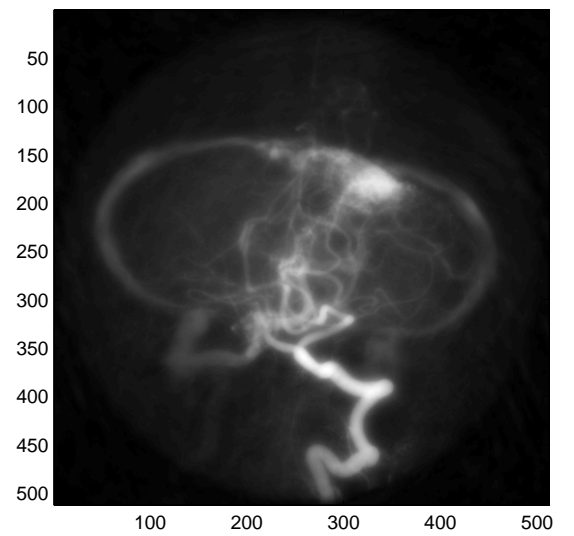

(b)

Figure 1. Illustration of the NFS data set. (a) The sampling positions in k-space for the first two spiral trajectories. (b) A reconstruction averaged over all coils and all time instants.

each consecutive set of 5 spirals, using a complex average in cases where two or more samples have the same neighbouring grid point. The resulting array is then transformed via the inverse 2D FFT to form an image. The under-sampling generates significant artifacts on each reconstruction, but the use of the temporal basis (described below) reduces these to an acceptable level.

\subsection{Combining data from coils}

The simulated data was supplied in the form of multiple recordings, simulating the use of an 8-coil receiver unit, as described above in Subsection 3.1. It was therefore possible to form 8 separate reconstructions, one for each "coil". It should however be possible to exploit the multiple recordings and knowledge of the individual coil sensitivities to improve the image reconstruction. In this case neither the SENSE or SMASH approaches seemed to offer much promise. SENSE requires a regular (patterned) form of under-sampling in order to apply a linear algebraic approach to lowering the effect of aliasing. SMASH only has the ability to recover regions of k-space immediately adjacent to the sampling points, which would have the effect of broadening the spiral trajectories, say to 3 or 5 pixels wide, instead of a single pixel wide. Observations of the corresponding sampling pattern point spread functions indicated that the effect on image quality would be relatively small. We adopted a simpler approach, therefore, as described below.

For each pixel in a given frame, a vector of complex amplitudes, $\mathbf{d}$, dimensioned $8 \times 1$, is generated by means of inverse Fourier transformation from the array of spiral samples. A set of relative coil sensitivities, $\mathbf{c}$, also dimensioned $8 \times 1$ and also expressed as complex amplitudes, is available a priori. We seek therefore to find the pixel magnitude $|f|$, according to the model

$$
\mathbf{d}=f \mathbf{c} .
$$

The MMSE solution to this is simply to minimise $|\mathbf{d}-f \mathbf{c}|^{2}$. In our implementation this has been encoded as

$$
f=\left|\frac{\sum_{m=1}^{8}\left(\mathcal{R}\left(c_{i}\right) \mathcal{R}\left(d_{i}\right)+\mathcal{I}\left(c_{i}\right) \mathcal{I}\left(d_{i}\right)\right)+j \sum_{m=1}^{8}\left(\mathcal{R}\left(c_{i}\right) \mathcal{I}\left(d_{i}\right)-\mathcal{I}\left(c_{i}\right) \mathcal{R}\left(d_{i}\right)\right)}{\sum_{m=1}^{8}\left|c_{i}\right|^{2}}\right|
$$

where $\mathcal{R}(x), \mathcal{I}(x)$ denote the real, imaginary parts of $x$, respectively. This form allows the relatively efficient vector and matrix operations of MATLAB (MathWorks, Natwick, Mass.) to be used. 


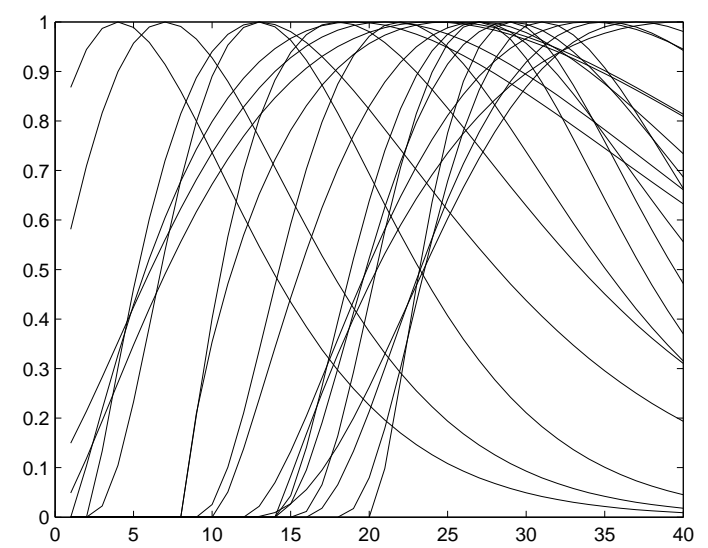

(a)

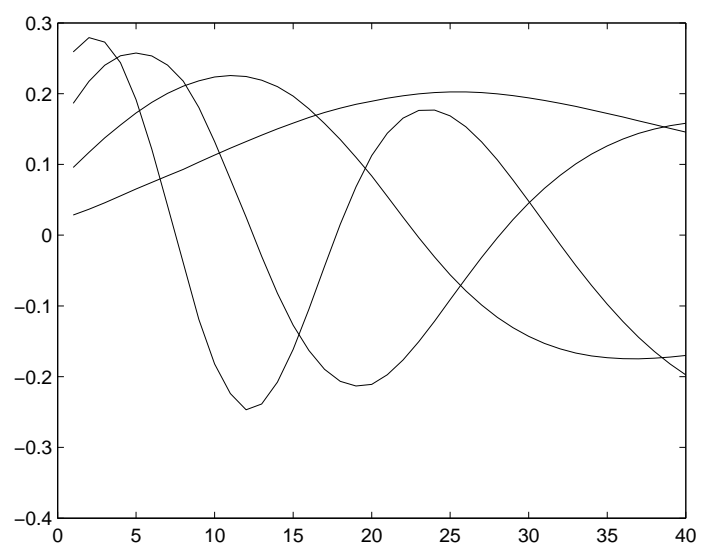

(b)

Figure 2. Illustration of the formation of a temporal basis set: (a) the first 20 out of a set of 100 unit amplitude gamma variate functions with randomly assigned parameters, and (b) the first 4 basis functions generated from the training set.

\subsection{Computing the temporal basis}

As described above in Subsection 2.4, a temporal basis was formed from a set of gamma variate functions $G$. The 200 spirals generated 40 frames at 5 spirals per frame, so $N=40$, representing a total interval of 10 seconds. A total of 100 functions were generated, so $G$ was $100 \times 40$. The first 20 of these functions are shown in Fig. 2(a). In each case the peak amplitude was fixed at unity while the three other parameters in Eq. 2 were randomly chosen with a uniform distribution from within the following ranges: $t_{0}-2$ to +5 seconds; $t_{\max }+2$ to +7 seconds; and $\alpha 0.8$ to 3.0. These ranges were chosen so that the 100 gamma variate functions represented a reasonable approximation to the expected shape of intensity-versus-time responses in the various parts of the FOV where blood vessels lay.

The algorithm detailed in Subsection 2.4 was then applied. Experimentation indicated that a total of 4 temporal basis functions was sufficient to fit the set of sample functions in $G$ within a small MSE, so the results presented in the next section are for 4 basis functions if not otherwise stated. Fig. 2(b) shows the four basis functions generated by the process. While recomputing the basis functions for a different set of gamma variate exemplars with the same parameter ranges will generate a different basis, experience shows that the functions are very similar in nature each time. Likewise, making small changes to the ranges of the gamma-variate parameters was found to have relatively little effect on the basis functions formed by KLT, indicating that the process is reasonably robust.

\section{RESULTS}

In Fig. 3 reconstructions of a single frame (the 10th out of a total of 40, with 5 spirals per frame and 4 frames per second) are shown for various methods. In all cases simple nearest-neighbour assignment of $\mathrm{k}$-space data onto the Cartesian grid was used, with no attempt to enforce the Hermitian property in k-space or to otherwise fill in the missing samples. Near the center of k-space, where several spiral samples may fall within a particular $\mathrm{k}$-space pixel, the complex average of the measurements was assigned.

A direct inverse transformation from single-coil data without any compensation for the coil sensitivity and without any account taken of temporal variation is shown in Fig. 3(a). Note the spiral-like artifacts; when the sequence of reconstruction is observed, the artifacts appear to swirl around the image. The effect of combining the data from all 8 coils, incorporating the sensitivity map information, is shown in Fig. 3(b). The edge of the circular FOV appears because the coil sensitivity maps were only defined out to that boundary. There is some slight improvement in the level of sampling artifacts, but the appearance of the blood vessels is still noticeably affected by the presence of artifacts generated by the severe under-sampling in k-space. 


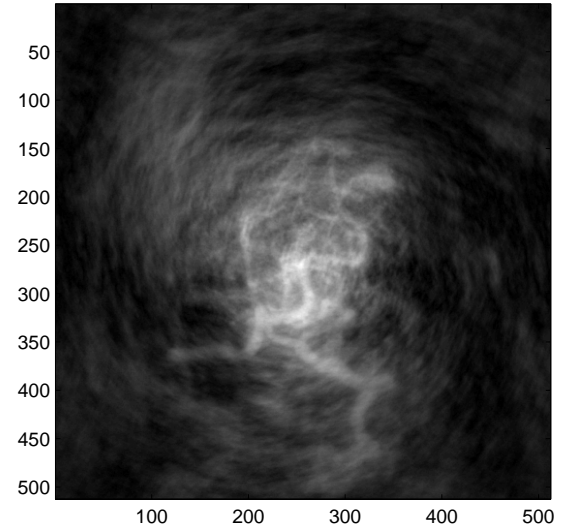

(a)

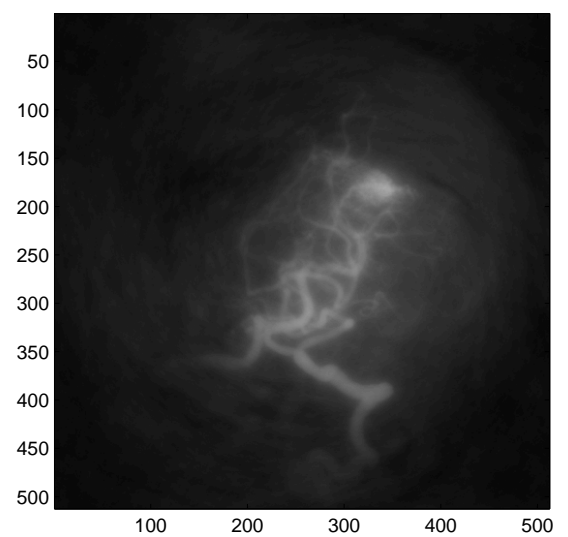

(c)

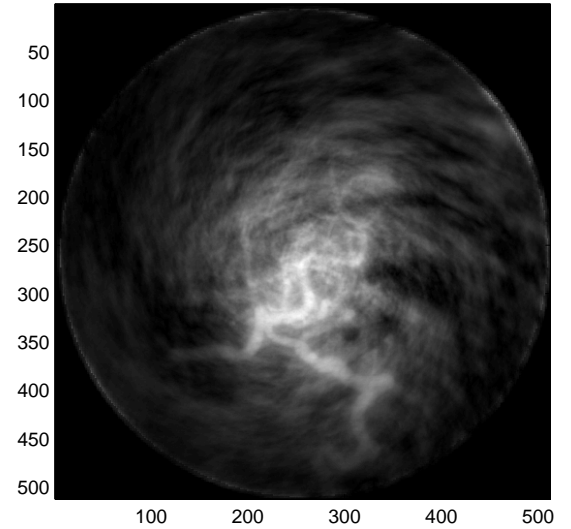

(b)

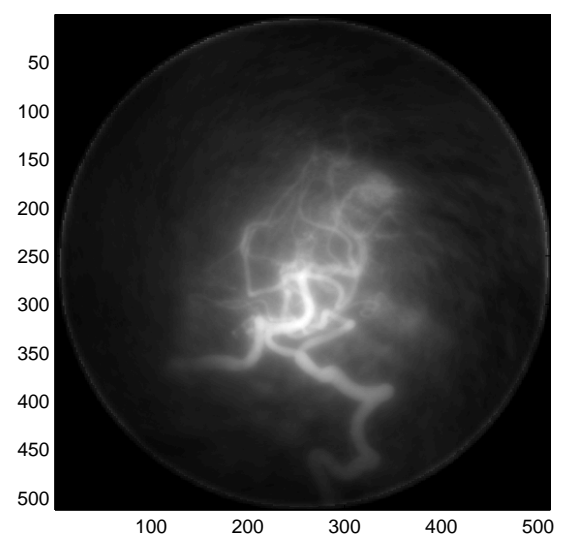

(d)

Figure 3. Reconstructions of frame 10 out of 40 with 4 frames per second and each frame generated from 5 spirals: (a) direct inverse transformation of k-space data for coil 1 only; (b) direct inverse transformation, but combining all coils with sensitivity adjustment; (c) applying a temporal basis with 4 basis functions for coil 1 only; and (d) applying a temporal basis with 4 basis functions, combining all coils with sensitivity adjustment. 


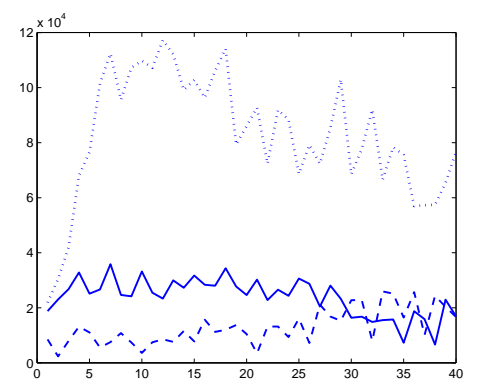

(a)

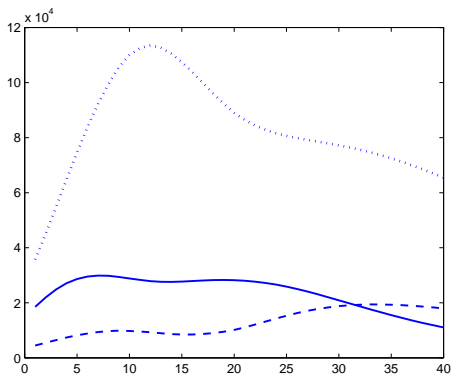

(b)

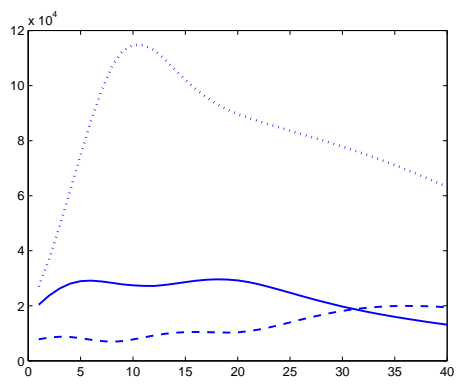

(c)

Figure 4. Study of the variation in reconstructed intensity with time within the vascular tree. Three small regions were segmented from the image: region $A$ is within the prominent artery lower center (solid curves); region $B$ is close to the center (dotted curves); and region $C$ is within the peripheral venous region upper left (dashed curves). (b) Intensity versus time curves generated by direct inverse transformation and combining all coils (i.e. without any temporal constraints). (b) Intensity versus time curves generated by applying the temporal basis (four basis functions only), combining all coils. (c) As for (b), but with 6 basis functions.

Corresponding reconstructions with a temporal basis applied are shown in Fig. 3(c) and (d). In both cases only the first 4 temporal basis functions, computed as explained above in Section 3, were used. In Fig. 3(c), the reconstruction for single-coil data without any compensation for the coil sensitivity is shown, while in Fig. 3(d), all coil data has been combined. In both cases the use of the temporal basis has had a dramatic effect in suppressing the background artifacts and, more significantly, improved the clarity of the vessels comprising the arterial tree greatly. The venous structure is not visible, since the frame is relatively early in the sequence.

Intensity versus time curves for selected regions within the FOV are shown in Fig. 4. Three small regions were segmented from the image (refer to Fig. 1(b)): region $A$ is within the prominent artery lower center, region $B$ is close to the center, and region $C$ is within the peripheral venous region on the left of the FOV. The mean intensity of pixels within each region was calculated frame-by-frame to generate the curves. Fig. 4(a) shows curves for regions $A, B$ and $C$ generated by direct inverse transformation and combining all coils (i.e. without any temporal constraints), while Fig. 4(b) shows curves generated by applying the temporal basis (four basis functions only), combining all coils. The smoothing effect of applying the basis is clearly evident. For comparison Fig. 4(c) shows the curves generated when an additional 2 basis functions are added; Figs. 4(b) and (c) are sufficiently similar to suggest that 4 basis functions are sufficient in this case.

Fig. 5 shows 5 frames selected from the sequence of 40 frames. The temporal basis of 4 basis functions has been used and data from all coils combined. Note that the general quality is reasonable and there is a clear progression in time throughout the sequence.

\section{DISCUSSION}

Results are presented above for a set of 4 temporal basis functions formed by KLT. The number was chosen as the minimum required to achieve a reconstructed sequence consistent with expectations for the set of data. In general, the basis needs to be tailored to the specific imaging situation. For example, there is a considerable difference between arterial and venous contrast injection, so the nature and number of basis functions need to be chosen accordingly. In addition, a different basis, for example one based on wavelets, may produce better results. Wavelets are well known to be good for representing transient signals and those which are expected to have similar shape. ${ }^{23}$

In the paper, results are presented for simulated spiral trajectory data with a relatively unsophisticated reconstruction method. The application of the temporal basis should be equally applicable to other sampling strategies and reconstruction methods and actual CE-MRA data. We plan to investigate the usefulness of the temporal basis with SENSE, ${ }^{2} \mathrm{SMASH}^{1}$ and our own algorithm for parallel MRI, GUISE. ${ }^{15}$ 


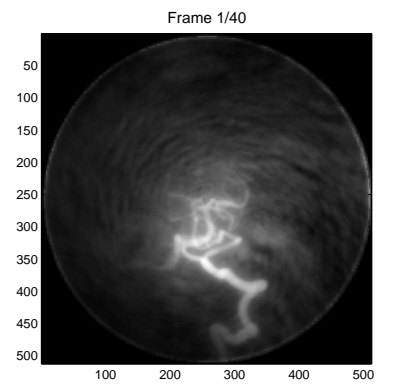

(a)

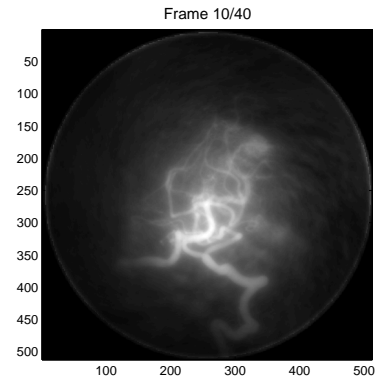

(b)

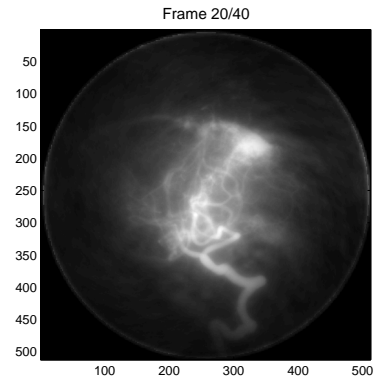

(c)

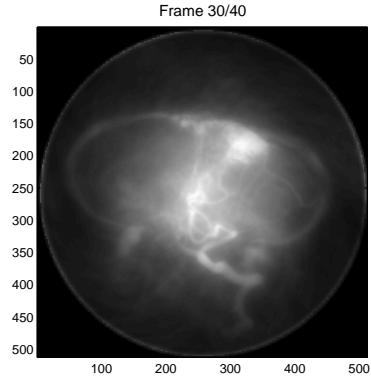

(d)

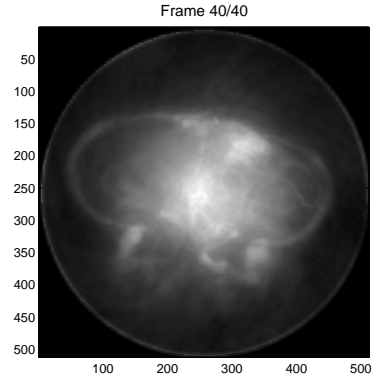

(e)

Figure 5. Reconstructions of five frames from a total sequence of 40 at 4 frames per second and each frame generated from 5 spirals: (a) frame 1; (b) frame 10; (c) frame 20; (d) frame 30; and (e) frame 40.

The key to virtually any imaging process is the SNR achieved in the collection of data and the reconstruction into an image. Generally in MRA there is a definite trade-off between SNR and time resolution. However, there is room to improve the SNR in a reconstructed sequence by exploiting more prior knowledge. We conjecture that the imposition of an appropriate temporal basis has the potential to improve SNR without sacrificing significant temporal resolution. We plan to extend the work presented here to include the analysis of SNR.

The object being imaged in CE-MRA is real so its Fourier transform is Hermitian. ${ }^{22}$ In principle this means that the samples obtained along any spiral trajectory have known conjugate duals reflected through the origin of k-space. While this suggests that k-space coverage is improved by invoking this prior knowledge, in practice the property may be of limited use. Experiments have shown that the image space point spread functions corresponding to the spiral sampling pattern with and without invoking the Hermitian property feature the same central impulse spanning approximately 7 pixels. The images formed can therefore be expected to have the same degree of sharpness. The periphery of the PSF is lowered in amplitude in the Hermitian version, which suggests a lowering of artifacts in the reconstructions. However it is important to note that the SNR is not improved, since one noisy measurement is being used to generate two k-space values.

\section{CONCLUSIONS}

The diffuse nature of the mixing of contrast agent and blood, coupled with the transient wash-in and wash-out during the recording of a CE-MRA sequence, suggest that each frame in a sequence is highly correlated to the frames next to it in time. A method is proposed which uses the prior knowledge that the gamma variate curve is a useful model for the image intensity versus time observed in locations where contrast-containing blood flow is present. The preliminary results presented suggest that considerable improvement in the quality of reconstructed sequences of images in CE-MRA can be achieved by incorporating an appropriate temporal basis. A novel approach employing the Karhunen-Loeve transform is described and results are presented for a publicly available simulated set of CE-MRA data. 


\section{REFERENCES}

1. Sodickson, D. and Manning, W., "Simultaneous acquisition of spatial harmonics (SMASH): fast imaging with radiofrquency coil arrays," Mag. Res. Med. 38(4), 591-603 (1997).

2. Pruessmann, K., Weiger, M., Scheidegger, M., and Boesiger, P., "SENSE: Sensitivity encoding for fast MRI," Mag. Res. Med. 42(5), 952-962 (1999).

3. Sodickson, D. and McKenzie, C., "A generalized approach to parallel magnetic resonance imaging," Med. Phys. 28(8), 1629-1643 (2001).

4. Insko, E. and Carpenter, J., "Magnetic resonance angiography," Seminars in Vasc. Surg. 17(2), 83-101 (2004).

5. Zhang, H., Maki, J., and Prince, M., "3D contrast-enhanced MR angiography," J. Mag. Res. Imag. 25, 13-25 (2007).

6. Aggarwal, N., Bandyopadhyay, S., and Bresler, Y., "Spatio-temporal modeling and adaptive acquisition for cardiac MRI," in [Proc. IEEE Int. Conf. Biomed. Eng.], 628-631 (2004).

7. Wierzbicki, M., Guiraudon, G., Jones, D., and Peters, T., "Dose reduction for cardiac CT using a registration-based approach," Med. Phys. 34(6), 1884-1895 (2007).

8. Madore, B., Glover, G., and Pelc, N., "Unaliasing by fourier-encoding the overlaps using the temporal dimension (UNFOLD), applied to cardiac imaging and fMRI," Mag. Res. Med. 42, 813-828 (1999).

9. Maki, J., Prince, M., Londy, F., and Chenevert, T., "The effects of time varying intravascular signal intensity and k-space acquisition order on three-dimensional MR angiography image quality," J. Mag. Res. Imag. 6, 642-651 (1996).

10. Prince, M., Chenevert, T., Foo, T., Londry, F., Ward, J., and Maki, J., "Contrast-enhanced abdominal MR angiography: optimization of imaging delay time by automating the detection of contrast material arrival in the aorta," Radiology 203, 109-114 (1997).

11. Korosec, F., Frayne, R., Grist, T., and Mistretta, C., "Time-resolved contrast-enhanced 3D MR angiography," Mag. Res. Med. 36, 245-351 (1996).

12. Bones, P., Alwesh, N., Connolly, T., and Blakeley, N., "Recovery of limited-extent images aliased because of spectral undersampling," J. Opt. Soc. Amer. A 18, 2079-2088 (2001).

13. Blakeley, N., Bones, P., Millane, R., and Renaud, P., "Efficient frequency-domain sample selection for recovering limited-support images," J. Opt. Soc. Amer. A 20, 67-77 (2003).

14. Gao, Y. and Reeves, S., "Optimal k-space sampling in MRSI for images with a limited region of support," IEEE Trans. Med. Imag. 19(12), 1168-1178 (2000).

15. Wu, B., Millane, R., Watts, R., and Bones, P., "Improved matrix inversion in image plane parallel MRI," Mag. Res. Imag. 27, 942-953 (2009).

16. Niendorf, T. and Sodickson, D., "Parallel imaging in cardiovascular MRI: methods and applications," NMR in Biomed. 19, 325-341 (2006).

17. Davenport, R., "The derivation of the gamma-variate relationship for tracer dilution curves," J. Nucl. Med. 24, 945-948 (1983).

18. Madsen, M., "A simplified formulation of the gamma variate function," Phys. Med. Biol. 37(7), 1597-1600 (1992).

19. Benner, T., Heiland, S., Erb, G., Forsting, M., and Sartor, K., "Accuracy of gamma-variate fits to concentration-time curves from dynamic susceptibility-contrast enhanced MRI: influence of time resolution, maximal signal drop and signal-to-noise," Mag. Res. Imag. 15(3), 307-317 (1997).

20. Rausch, M., Scheffler, K., Rudin, M., and Radu, E., "Analysis of input functions from different arterial branches with gamma variate functions and cluster analysis for quantitative blood volume measurements," Mag. Res. Imag. 18, 1235-1243 (2000).

21. Lee, T.-L., "CT perfusion: principles, applications and problems," in [Proc. SPIE], 5535, 77-88, SPIE (2004).

22. Jain, A., [Fundamentals of Digital Image Processing], Prentice-Hall, New Jersey (1989).

23. Mallat, S., [A Wavelet Tour of Signal Processing], Academic Press, London, 2nd ed. (1999). 